\title{
Human and pigeon suboptimal choice
}

\author{
Margaret A. McDevitt ${ }^{1}$ - James W. Diller ${ }^{2}$ - Malvina O. Pietrzykowski ${ }^{2}$
}

Published online: 19 August 2019

(C) The Psychonomic Society, Inc. 2019

\begin{abstract}
Many studies have shown that pigeons will sometimes behave suboptimally by choosing an option that provides food less frequently over one that provides food more frequently. The critical factor in driving suboptimal behavior in these procedures is that the delayed outcomes are differentially signaled on the suboptimal alternative, but not the optimal alternative. Although this procedure is frequently cited as potentially analogous to human gambling, there is little empirical data to evaluate this assertion. The present study tested both pigeon (Experiment 1) and human (Experiment 2) subjects with a suboptimal choice task. Subjects chose between a suboptimal alternative that provided a large reinforcer $20 \%$ of the time and an optimal alternative that always provided a small reinforcer. Stimuli presented during the delays signaled the outcomes on the suboptimal alternative in some conditions. When outcomes were signaled, pigeons chose the suboptimal alternative more frequently than did humans. When the outcomes were not signaled, pigeons' choices became more optimal, but humans' choices did not. Humans' suboptimal choice was unrelated to performance on a probability discounting task. Overall, these findings suggest that although both pigeons and humans can choose suboptimally, more research is needed in order to determine whether non-human performance on this task can serve as a model for human gambling.
\end{abstract}

Keywords Choice $\cdot$ Suboptimal behavior $\cdot$ Gambling $\cdot$ Preference $\cdot$ Key peck $\cdot$ Pigeons $\cdot$ Humans

\section{Introduction}

Pigeons, like other animals, typically show preferences that can be described as orderly and in tune with the available resources, i.e., they select options that provide richer schedules of reinforcement over leaner schedules (Herrnstein, 1970). Behavior reliably deviates from this pattern, however, in some circumstances. For example, in the suboptimal choice task, pigeons will demonstrate what appears to be suboptimal behavior when a lower reinforcement alternative provides informative signals that indicate which outcome is scheduled to occur at the end of the trial (for reviews, see McDevitt, Dunn, Spetch, \& Ludvig, 2016; Zentall, 2016b). In the original procedure (Kendall, 1974), pigeons chose between an optimal alternative that always ended with food after a delay and a suboptimal alternative that ended in food only half the time

Margaret A. McDevitt

mmcdevit@mcdaniel.edu

1 Department of Psychology, McDaniel College, 2 College Hill, Westminster, MD 21157, USA

2 Department of Psychological Science, Eastern Connecticut State University, Willimantic, CT, USA after an equivalent delay. When the food/no food outcomes on the suboptimal alternative were differentially signaled during the delay, pigeons chose the suboptimal alternative much more frequently than when that alternative did not provide signals. Although other features of the procedure are important in attenuating or magnifying the degree of suboptimal choice (e.g., Dunn \& Spetch, 1990; McDevitt, Spetch, \& Dunn, 1997; Spetch, Belke, Barnet, Dunn, \& Pierce, 1990), the phenomenon depends on the presence of signals. In other words, choice of the suboptimal alternative is reduced if it does not provide differential signals or if a probabilistic optimal alternative provides differential signals.

Since Kendall's (1974) initial demonstration, suboptimal choice has been extensively studied in pigeons (see McDevitt et al., 2016 and Zentall, 2016b), and has been observed in monkeys (e.g., Blanchard, Hayden, \& BrombergMartin, 2015; Bromberg-Martin \& Hikosaka, 2009; Smith, Beran, \& Young, 2017) and starlings (e.g., Vasconcelos, Monteiro, \& Kacelnik, 2015). The procedure has also been used with rats, but the results have been less consistent (e.g., Mazur, 2007; Trujano \& Orduna, 2015; but see Cunningham \& Shahan, 2019). Human participants, however, have received little empirical attention despite the suggestion that the suboptimal choice task "provides a reasonable analog to human commercial gambling behavior" (Zentall, 2016a, p. 
110, cf. Zentall, 2016b; Zentall \& Laude, 2013; Zentall, 2014).

Despite the potential importance of an application to human gambling, only one basic laboratory study has used the suboptimal choice procedure with human subjects (Molet et al., 2012). In this study, college students were presented with a game-like task in which they were instructed to kill alien generals by clicking on spaceships with a computer mouse. The contingencies were arranged to correspond to a procedure used by Zentall and Stagner (2011) with pigeons. In the pigeon study, choice of a suboptimal alternative led to a delay that usually ended with no food delivery, but sometimes led to a large reinforcer (ten pellets). As in the previous suboptimal choice studies, the stimulus presented during the delay signaled which outcome would occur. Choice of the other, optimal alternative led to a delay that always ended with a small (three pellets) reinforcer. Pigeons, on average, preferred the suboptimal alternative. However, when the outcomes of the suboptimal alternative were no longer signaled by the delay stimuli (i.e., each stimulus led to the same probability of ten pellets), the pigeons, on average, reversed their preference.

In Molet et al.'s (2012) study, spaceships were presented within two planetary systems (indicated by the words "ARTO" or "ZORB"), and the background screen color and landscape corresponded to different alternatives. After selecting a planetary system and destroying spaceships, a message appeared that indicated how many generals were killed during the visit (which served as the reinforcer). Each alternative was associated with a variable magnitude of reinforcers. The optimal alternative always led to the destruction of $3 \pm 1$ generals, regardless of the background color. On $20 \%$ of presentations of the suboptimal alternative, a high number of generals were killed when the background was one color (10 \pm 1 ), but no generals were killed on the other $80 \%$ of the trials in which the background was a different color. Participants were selected based on their responses to a survey in which they reported how often they engaged in activities related to gambling, so that one group consisted of non-gamblers and the other group consisted of frequent (i.e., weekly or daily) gamblers. The results showed that gamblers selected the suboptimal alternative more frequently $(56.5 \%$ of the choice trials) than the non-gamblers $(23.0 \%)$. Thus, the non-gamblers preferred, on average, the optimal alternative.

Although Molet et al.'s (2012) study demonstrates that human participants will sometimes choose a suboptimal alternative over an optimal alternative, we do not know if that choice occurs for the same reason as in nonhuman animals. That is, Molet et al.'s study included a suboptimal alternative that always provided signals, and thus it is unclear whether suboptimal choice would have decreased if the signals were removed (i.e., if the colored backgrounds no longer were differentially associated with the number of generals killed), or if any change in preference would be associated with frequency of gambling. As noted above, pigeons in this procedure will reliably choose the optimal alternative when the suboptimal alternative does not provide differential signals, as shown by Zentall and Stagner (2011), but the importance of the signals was not assessed in Molet et al.'s study.

The role of signals has been evaluated in an applied study in which children were given choices between a suboptimal alternative (sometimes with signaled outcomes, sometimes without) and an optimal alternative (Lalli, Mauro, \& Mace, 2000; see also Lalli \& Mauro, 1995). Two children with developmental disabilities were more likely to choose a suboptimal alternative when it provided signals than when it did not (Lalli et al., Experiment 1) in a procedure modeled after the one used with pigeons. A similar result was shown with clinically relevant behaviors (Experiment $3, \mathrm{~N}=1$ ). In addition, choice behavior was influenced by the delay duration (Experiment 1, N=2), consistent with results with pigeons (Dunn \& Spetch, 1990; Spetch et al., 1990).

Thus, despite its potential importance in understanding choice behavior, few empirical investigations of the suboptimal choice task have included humans. The present study extended Molet et al.'s (2012) work by employing the same suboptimal choice procedure (also based on Zentall \& Stagner, 2011) with both pigeons and humans, but included unsignaled comparison conditions absent in the prior research. Experiment 1 presents the study with pigeons and Experiment 2 presents the study with human participants to compare the degree to which suboptimal choice occurs and is influenced by the presence of signals.

\section{Experiment 1}

\section{Method}

Subjects The subjects were ten adult ex-racing pigeons with experience in a variety of experimental procedures and were cared for in accordance with McDaniel College's Animal Care Guidelines. They were maintained at approximately $85 \%$ of their free-feeding weights by grain obtained during experimental sessions and immediate post-session feedings when necessary. The pigeons were housed in individual cages under a 12-h light/dark cycle, with water and grit freely available.

Apparatus Two operant chambers (approximately $360 \mathrm{~mm}$ wide, $320 \mathrm{~mm}$ long, and $350 \mathrm{~mm}$ high) were used. Three translucent response keys, $25 \mathrm{~mm}$ in diameter, were mounted on the front intelligence panel $260 \mathrm{~mm}$ above the floor and $72.5 \mathrm{~mm}$ apart. The center key was never used in these experiments. Each side key could be illuminated from the rear by standard IEE 28-V 12-stimulus projectors. A 28-V 1-W miniature lamp, located $87.5 \mathrm{~mm}$ above the center response key, provided general chamber illumination for the duration of 
each session. Directly below the center key and $95 \mathrm{~mm}$ above the floor was an opening ( $57 \mathrm{~mm}$ high by $50 \mathrm{~mm}$ wide) that provided access to a solenoid-operated grain hopper filled with mixed grain. When activated, the food hopper was raised for $4 \mathrm{~s}$ and illuminated from above with white light by a $28-\mathrm{V}$ 1-W miniature lamp. A computer and a MED-PC interface, located in an adjacent room, controlled experimental events.

Procedure The basic procedure is shown in Fig. 1. The optimal alternative was presented on one side key and consisted of a circle stimulus that, when pecked once, was replaced with a terminal-link (TL) stimulus (e.g., green or red key-light). One stimulus was presented on $20 \%$ of the trials and the other was presented on the remaining $80 \%$ of the trials. Regardless of which stimulus was presented, it remained illuminated for $10 \mathrm{~s}$ and was followed by 3 -s access to the food hopper.

The suboptimal alternative was presented on the opposite side key, and also consisted of a circle stimulus, which when pecked once, was replaced with a TL stimulus (e.g., blue or white keylight). In the SIG condition (shown in the top panel of Fig. 1), one stimulus (e.g., blue) was presented on $20 \%$ of the trials, and after $10 \mathrm{~s}$ always terminated with 10 -s access to the food hopper. The other stimulus (e.g., white) was presented on the remaining $80 \%$ of the trials, and after $10 \mathrm{~s}$ always terminated with a 10 -s blackout. In the UNSIG condition (shown in the bottom panel of Fig. 1), the key-lights were presented with the same probabilities as in the SIG condition, but each led to 10 -s access to food $20 \%$ of the time and blackout $80 \%$ of the time.

The side location of the alternatives and the stimulus assignments were counterbalanced across subjects. Five subjects experienced the SIG condition first (Group S-U) and five subjects experienced the UNSIG condition first (Group U-S). After 16 sessions were completed, the conditions were switched between the two groups, and an additional 21 sessions were completed in the second condition. The TL stimuli and side assignments remained the same during the second condition.
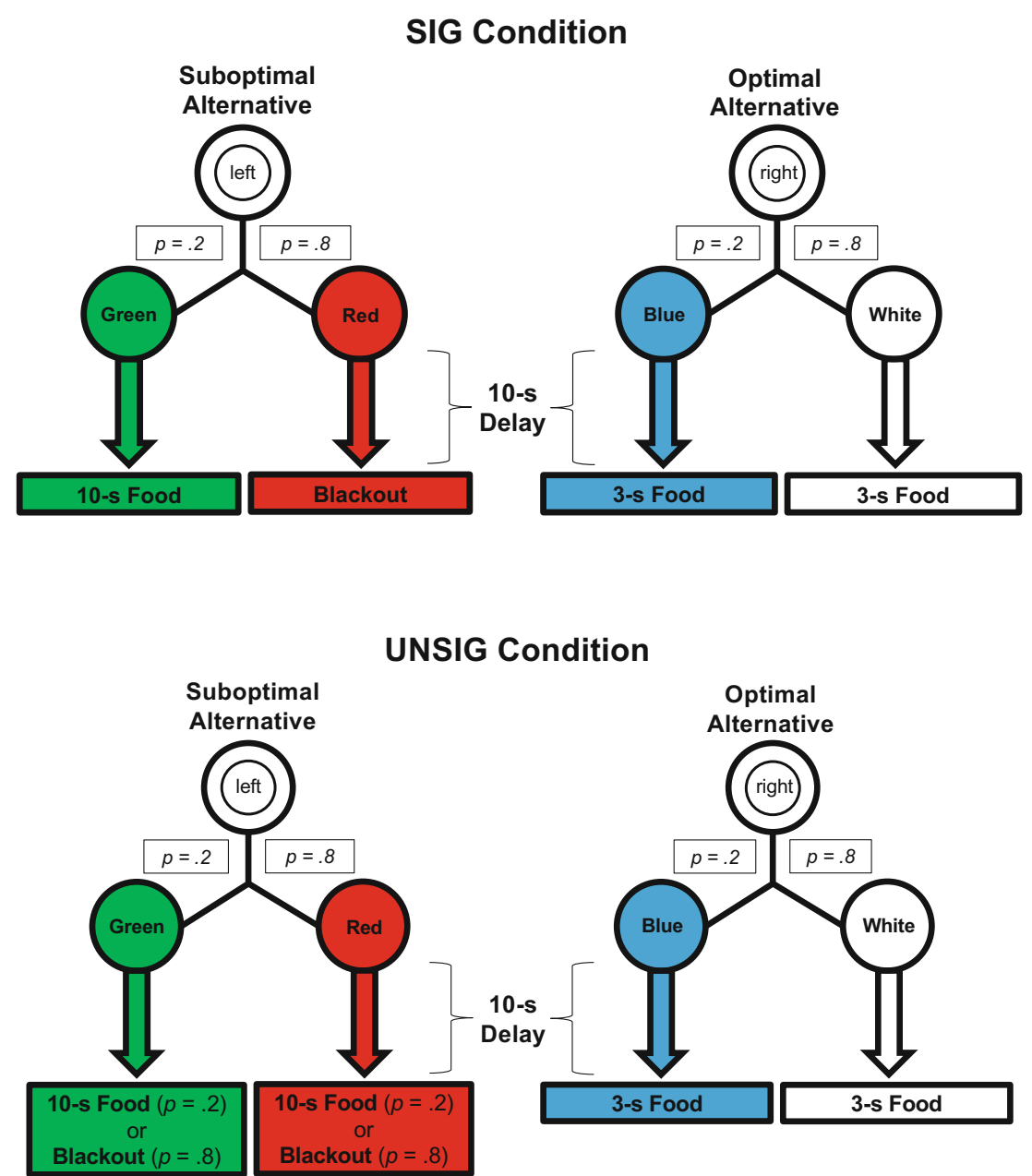

Fig. 1 Suboptimal choice procedure in which the optimal alternative always provided 3 -s access to food and the suboptimal alternative provided 10 -s access to food on $20 \%$ of the trials. In the SIG condition, keylight colors in the terminal links were correlated with outcomes on the suboptimal alternative. A single peck was required in the initial link, and the terminal-link duration was $10 \mathrm{~s}$. The side and stimulus assignments were counterbalanced across subjects 
Each session lasted for 40 min and began with 20 forcedexposure (FE) trials in which only one alternative was presented. Half of the FE trials consisted of the suboptimal alternative and half consisted of the optimal alternative, presented in a randomized order. Following the FE trials, all subsequent trials presented a choice between the suboptimal and optimal alternatives (i.e., both circle stimuli were presented simultaneously). The intertrial interval was $10 \mathrm{~s}$.

\section{Results and discussion}

Means are reported with corresponding $95 \%$ confidence intervals (CIs) and all significant effects are reported at $p<.05$. Figure 2 shows the development of preference for the suboptimal alternative for the two groups of subjects across both conditions of Experiment 1. Overall, preference for the suboptimal alternative was more extreme when the outcomes were signaled $(M=0.62$, $95 \%$ CI $[0.45,0.78])$ than when they were not signaled $(M=$ $0.14,[-0.02,0.30])$. Statistical analysis revealed a significant main effect of signals, $F(1,8)=41.49, p<.01$. These results replicate the findings of Zentall and Stagner (2011), which showed that pigeons, overall, preferred a suboptimal alternative when the outcomes associated with it were signaled (Experiment 1) compared to other pigeons that preferred the optimal alternative, on average, when no differential signals were provided (Experiment 2). The present results are also consistent with other studies of suboptimal choice that demonstrate that the presence of signals is a key factor in suboptimal choice (McDevitt et al., 2016; Zentall, 2016b).

Figure 3 shows the mean choice proportions for each bird, averaged over the last four sessions of each condition. The difference between conditions is more apparent in the group that received the UNSIG condition first, but there was no significant interaction between condition order and signaling contingency $(p=.24)$.

\section{Experiment 2}

Experiment 2 extends the work of Experiment 1 by evaluating this paradigm with human participants. In this systematic replication of the study by Molet et al. (2012), an unsignaled condition was added to evaluate the influence of signals within this procedure. To assess the extent to which impulsivity relates to this type of choice (see Laude, Beckmann, Daniels, \& Zentall, 2011, for a demonstration of this relation in pigeons), our participants also completed a probability discounting task.

Probability discounting is the decrease in the value of a reinforcer as its certainty declines (cf. Rachlin, Raineri, \& Cross, 1991). In laboratory models of probability discounting, participants are often presented with a choice between two reinforcers. One is relatively small and delivered with high certainty while the second is larger and delivered with lower

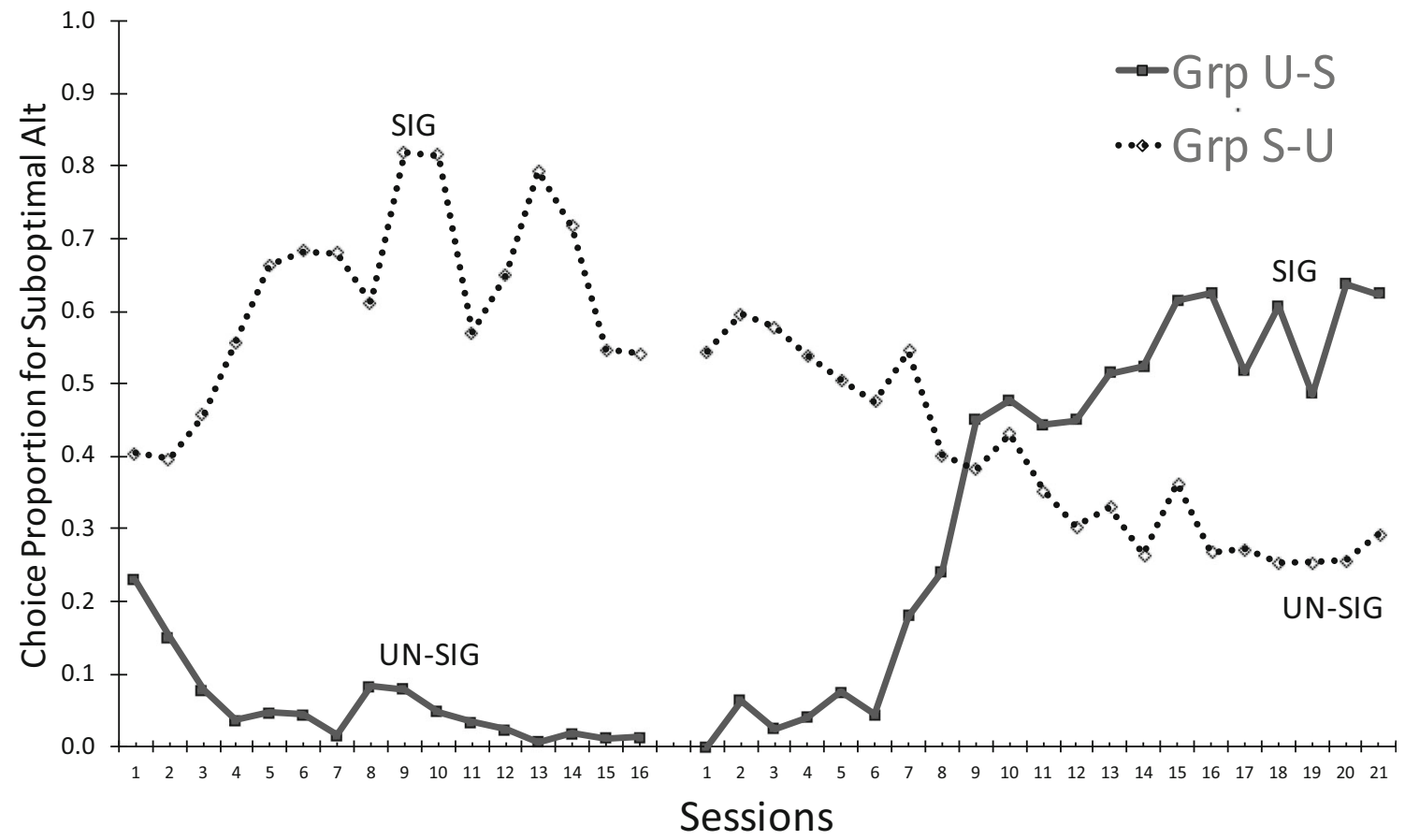

Fig. 2 Mean choice proportions for the suboptimal alternative for the two groups of birds in Experiment 1. In one group, the outcomes on the suboptimal alternative were signaled (SIG) in the first condition and not signaled (UNSIG) in the second condition (Grp S-U). The other group (Grp U-S) received the conditions in the reverse order 




SIG

UN-SIG

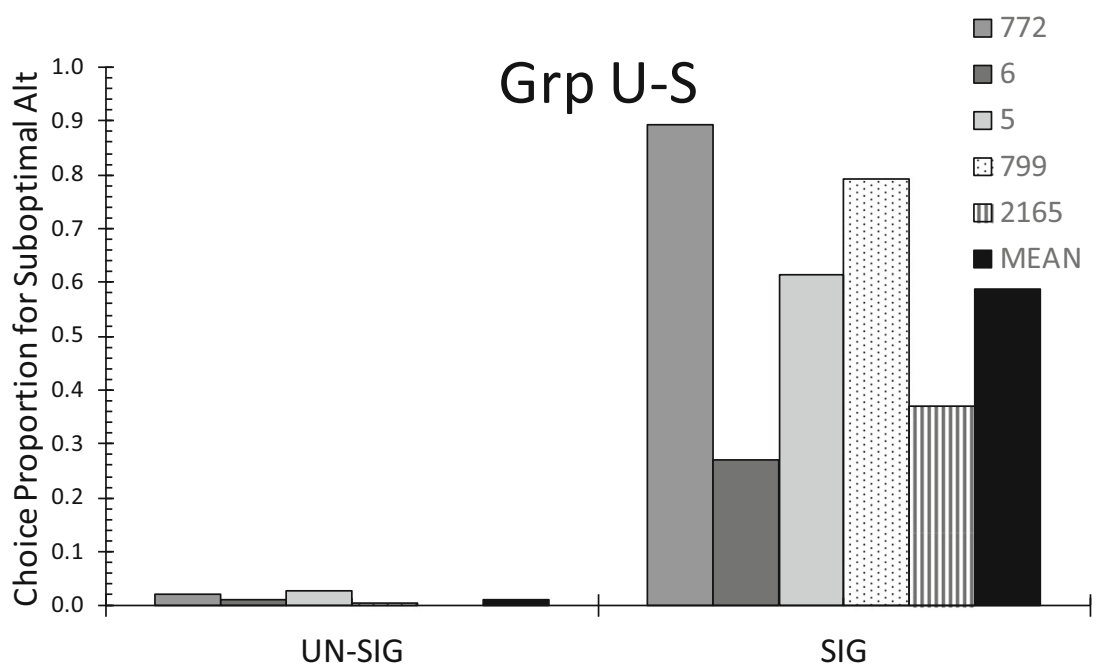

Fig. 3 Mean choice proportions for the suboptimal alternative for each bird in Experiment 1 in conditions in which the suboptimal alternative was signaled (SIG) or unsignaled (UNSIG). Means are the average of the last four sessions in each condition

certainty. The selection of the larger, less certain reinforcer has been defined as a self-controlled choice, and the selection of the smaller, guaranteed reinforcer is an impulsive choice. Individuals discount probabilistic reinforcers at varying rates, and probability discounting has been implicated in the development of pathological gambling (e.g., Petry, 2012).

\section{Method}

Participants and apparatus Participants $(\mathrm{N}=60)$ were undergraduate students over the age of 18 years who were recruited from the subject pool of the Department of Psychological Science at Eastern Connecticut State University. Data from one male participant were excluded from analyses due to a computer error, leaving a final total of 59 participants; 50 were female. These participants were randomly assigned to one of four conditions: signaled only ( $n=16$, five male), unsignaled only $(n=15)$, signaled first ( $n=14$, four male), or unsignaled first $(n=14)$. Previous research in this area (Molet et al., 2012) was used to guide the target sample size, and the sample in the current study is larger than the sample size in this prior work ( $n=10$ in each of the two groups in Experiment 1). Testing each of our 59 participants in the suboptimal choice task twice (as described below) further increased the sensitivity of the present study.

Experimental tasks were presented on a Dell laptop running Windows10. Experimental events were programmed using PsychoPy version 1.83.04 (Peirce, 2007) by the first author. Sessions occurred individually, with a researcher and experimenter seated at a table in a large room.

Procedure All procedures for Experiment 2 were approved by Eastern Connecticut State University's Committee on the Use of Human Subjects in Research. Prior to completing the laboratory tasks described here, participants also completed online surveys, including the South Oaks Gambling Screen (Lesieur \& Blume, 1987).

The suboptimal choice procedure was similar to that in Experiment 1 (as shown in Fig. 1), but modified to accommodate 
human subjects (see Fig. 4). Points were used as a putative reinforcer instead of access to mixed grain. And, instead of responding on keys in an operant conditioning chamber, participants responded via a mouse click in a simple game-like environment modeled after the work of Molet et al. (2012).

A computer program presented the participants with choices between a suboptimal alternative and an optimal alternative. Each trial began with the presentation of one or two images of doors (in forced-exposure [FE] or choice trials, respectively) that led to a 10-s presentation of a colored circle followed by a 10-s display of a message about the number of points that were won (i.e., "You got nothing," "You WON 3 points!" or "You WON 10 points!!!") and a 4-s intertrial interval, during which only the cumulative point total was displayed.

Each session consisted of a series of $20 \mathrm{FE}$ trials followed by 20 choice trials. In each FE trial, a single white door was shown on the right or left side of the screen. Depending on the location of the door on the screen (left or right), clicking on it with the computer mouse produced one of two sets of consequences. One side (the suboptimal choice) was associated with earning 10 points $20 \%$ of the time, or 0 points $80 \%$ of the time. The other side (the optimal choice) always yielded 3 points. In the choice trials, both doors were concurrently available. When the doors were presented at the start of each trial, the cursor was repositioned to the center of the screen. The program ended following 20 choice trials. A cumulative total of points won was always presented below the other stimuli (doors, circle, and text). Colors and the sides correlated with the suboptimal and optimal choices were counterbalanced across participants.

Broadly, there were two versions of the computer program: one with signals (i.e., correlated colored stimuli associated with each outcome) and one without signals (i.e., regardless of which outcome was scheduled, a black circle was presented). Each participant completed the suboptimal choice task twice, with the probability discounting task (described below) between the administrations. Participants were randomly assigned to one of four conditions: (1) signaled only, (2) unsignaled only, (3) signaled first, and (4) unsignaled first.
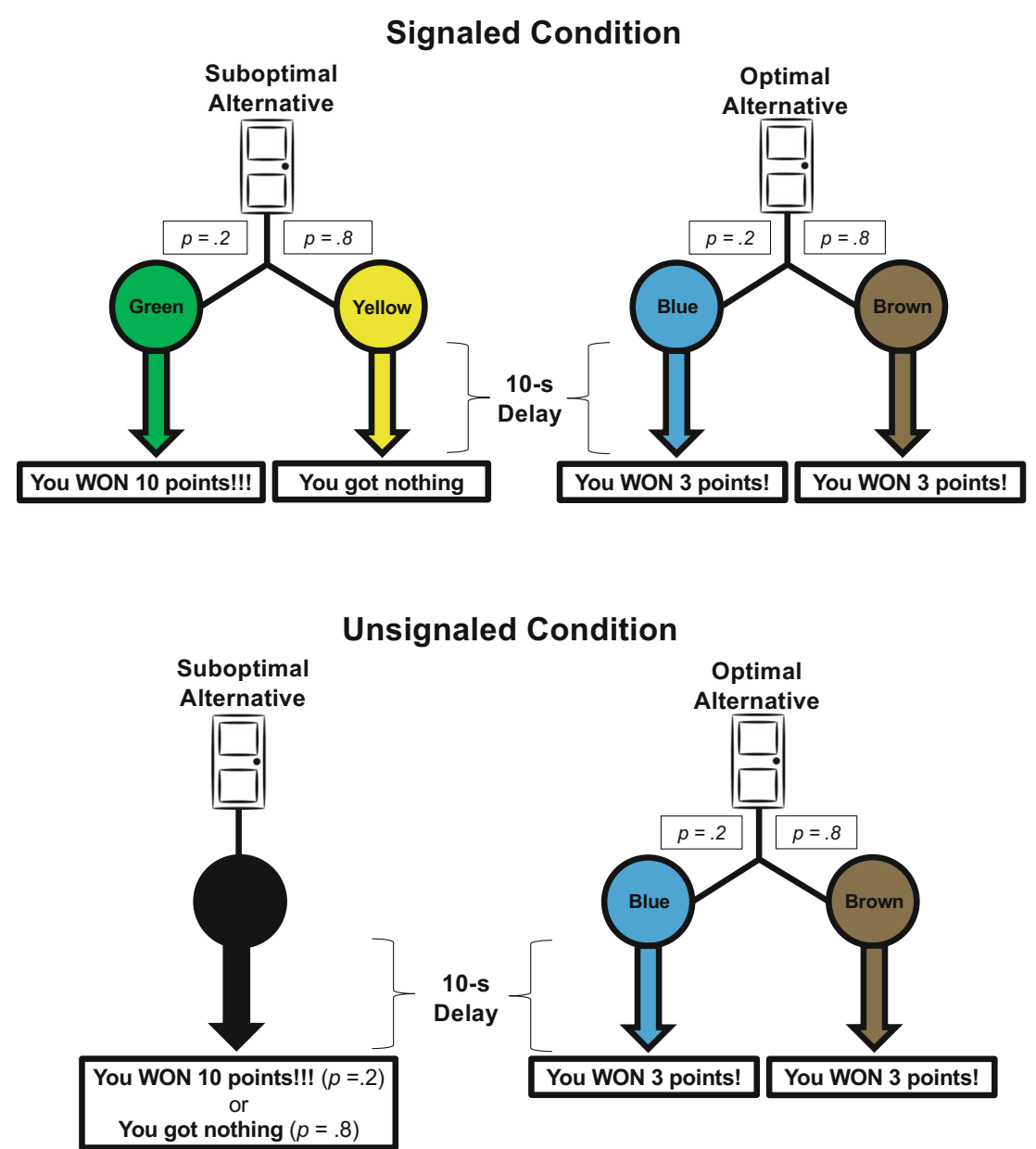

Fig. 4 Suboptimal choice procedure in which the optimal alternative always provided 3 points and the suboptimal alternative provided points on $20 \%$ of trials. In the signaled condition, colors in the terminal links were correlated with outcomes on the suboptimal alternative. A single

peck was required in the initial link, and the terminal-link duration was 10 s. The side and stimulus assignments were counterbalanced across subjects 
Participants in the "only" conditions experienced the same program (signaled or unsignaled) twice.

Prior to beginning the first session of the choice task, participants were told that they would be presented with pictures of doors on the computer screen, and that using the mouse to click on the doors would earn them points. They were instructed to earn as many points as possible.

Probability discounting task Probability discounting was assessed using an analog choice task (Rachlin et al., 1991). Participants were presented with a series of choices between hypothetical amounts of money (range: \$1-\$1,000) presented at hypothetical probabilities (ranging from $100 \%$ to $5 \%$ ) on a series of index cards. Based on the choices the participants made, an indifference point (i.e., the value at which the probabilistic and guaranteed amounts of money have equal subjective value) was derived for each probability and the indifference points were used to calculate area under the curve (AUC; Myerson, Green, \& Warusawitharana, 2001).

\section{Results and discussion}

Participants consistently preferred the optimal choice, with approximately $74 \%$ of all choice responses allocated to this alternative. Selection of the suboptimal alternative was consistent when it was signaled $(M=0.26,95 \%$ CI $[0.24,0.27])$ and unsignaled $(M=0.27,95 \%$ CI $[0.23,0.30])$. The mean proportions of suboptimal choice for each condition are presented in Fig. 5. As evident in this figure, results did not significantly differ among the four condition orders, indicating that suboptimal choice was not affected by the presence of discriminative signals. It is noteworthy that these values are close to the mean suboptimal choice for the non-gamblers (0.23) in Experiment 1 by Molet et al. (2012). The similarity in the degree of suboptimal choice in the present study and the prior work is consistent with the view that, despite some differences, the two procedures functioned similarly.

The mean AUC value obtained from the probability discounting task was 0.45 (95\% CI [0.39, 0.52]). There were no correlations between AUC and proportion suboptimal choice in either signaled $\left(r_{\mathrm{s}}(43)=.14, p=.37\right)$ or unsignaled $\left(r_{\mathrm{s}}(43)=.05, p=.77\right)$ conditions. Thus, the measure of impulsivity did not seem to be related to suboptimal choice. This failure to find a relation between these variables is interesting in light of the difference between gamblers and nongamblers in the study by Molet et al. (2012), in which gamblers were more likely to make the suboptimal choice than non-gamblers. In our study, we did not select participants for their gambling behavior, and the majority were non-gamblers (according to the South Oaks Gambling Screen), with only two participants indicating that their gambling was at a level similar to the "gamblers" in the study by Molet et al. Future research could directly evaluate both probability discounting and gamblinglike performance in a task similar to the one used in the present study (with and without signals) in gambling and nongambling participants.

\section{General discussion}

The current studies extend the work of Zentall and Stagner (2011) and Molet et al. (2012) by evaluating the effects of signals across human and nonhuman subjects. Similar to Zentall and Stagner's results, pigeons in Experiment 1 preferred, on average, the optimal alternative when the outcomes were not signaled but preferred the suboptimal alternative when the outcomes were signaled. In contrast, human participants in Experiment 2 consistently preferred the optimal, less

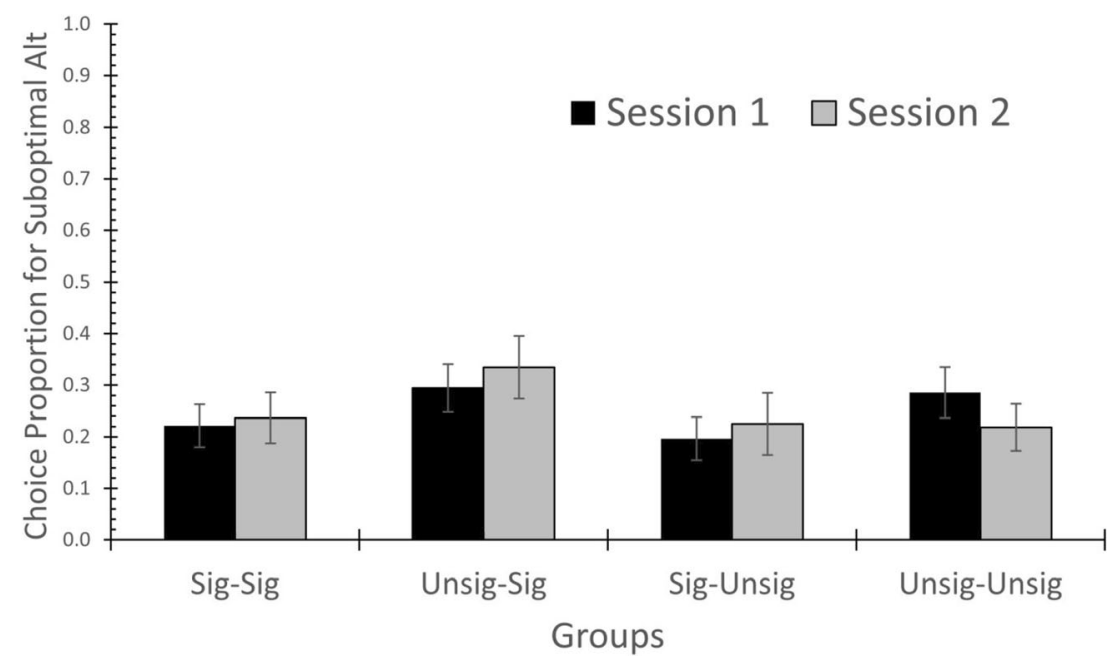

Fig. 5 Mean proportion of suboptimal choices in Experiment 2, by condition. The black bars represent the first session and the gray bars represent the second session. Each pair of bars represents a different

order of presentation of signaled and unsignaled conditions. Error bars represent standard error of the mean 
risky choice, regardless of whether or not the suboptimal alternative provided differential signals. Thus, signals influenced the pigeons' choice behavior, but not that of the human participants.

The procedure used in Experiment 2 of the present study closely approximated the procedure with pigeons used by Zentall and Stagner (2011), but awarded points rather than food. In comparison, participants in Molet et al.'s (2012) study were provided a message representing the number of generals killed within the context of a game with more detailed visual cues and instructions. Despite these differences, the degree of suboptimal choice observed in Experiment 2 of the present study replicates the results observed with non-gamblers in the study by Molet et al. In both studies, approximately onequarter of choices were made to the suboptimal alternative when it provided differential signals; the rate at which our participants engaged in the suboptimal choice was consistent across conditions. Thus, the present work replicates the previous results with humans, but with a larger number of subjects, none of whom were selected based on gambling behavior. Although our sample did not include as many gamblers as in Molet's study, so few studies have been conducted with humans in this procedure that it is important to establish a general baseline of behavior, which can aid in future assessment of subpopulations of humans such as frequent gamblers.

Relative to previous research, Experiment 2 also included additional conditions in which the signaling contingencies were either maintained over the two sessions or altered. When the suboptimal alternative provided stimuli uncorrelated with the trial outcomes, participants in the present study still chose it approximately one-quarter of the time. Thus, it remains uncertain whether suboptimal choices by humans and pigeons are similarly influenced by the signaling contingencies. However, despite not directly measuring whether participants explicitly detected the colors that signaled the outcomes, the stable response pattern suggests robust control by the contingencies of reinforcement in effect for our participants. Additionally, the present experiment counterbalanced sides within and between subjects, and involved easily discriminable stimuli on the suboptimal alternative (two colors signaled the different outcomes and a black stimulus was used when signals were not provided). Given that the presence of differential stimuli immediately following a suboptimal choice is the key variable in generating suboptimal preference with pigeons, it would be important to show that humans are similarly affected by the manipulation of the signals. Experiment 2 provided an initial step in this direction, but more work is needed: for example, assessment of signal sensitivity via a preference test between the colors associated with each option before and after sessions would provide evidence that the discrimination was learned.

One important variable may be the duration of the outcome delay. We selected a 10-s delay because that was the duration used by Zentall and Stagner (2011) and Molet et al. (2012), but future research should evaluate longer delay periods. Longer delays have been shown to enhance the degree of suboptimal behavior with pigeons (McDevitt, Pisklak, Spetch, \& Dunn, 2018; Spetch et al., 1990; Spetch, Mondloch, Belke, \& Dunn, 1994, Experiment 3). Interestingly, Lalli et al. (2000) found that children chose the suboptimal choice more frequently when signaled, but only in conditions in which the outcome delays were $30 \mathrm{~s}$ and, similar to the present study, did not show a reliable effect of signals with 10 -s delays.

One of the most intriguing findings shown in the study conducted by Molet et al. (2012) is that participants who reported regular gambling behavior demonstrated significantly greater suboptimal choice than those who did not gamble. In comparison to Zentall and Stagner's (2011) pigeons, the human gamblers in the Molet et al. study were more restrained, selecting the suboptimal alternative $56.5 \%$ of the time compared to $82.2 \%$ by the pigeons. Our pigeons were more similar to Molet et al.'s gamblers, selecting the signaled suboptimal alternative $62 \%$ of the time in Experiment 1, and our human participants behaved similarly to Molet's non-gambling participants. Figure 6 shows the mean results from all three studies, which used the same general procedure.

We tested for, but did not find, a correlation between scores on the probability discounting task and suboptimal choice task. We also did not observe an association between selfreported gambling behavior and suboptimal choice. It should be noted, however, that the number of participants in our Experiment 2 was more than double the number in the initial study by Molet and colleagues, so the lack of a finding was likely not due to a lack of statistical power. Zentall (2014) suggested that "this suboptimal choice task can be thought of as diagnostic of gambling behavior" (p.161), and probability discounting has been implicated in pathological gambling (cf. Petry, 2012). The lack of this association may be due to the characteristics of our sample. Only two of our participants received a score indicating "probable pathological gambler" on the South Oaks Gambling Screen (Lesieur \& Blume, 1987), and thus the lack of correlation may not be surprising. Given that very few of our human participants gambled and that Molet et al. found differences between regular gamblers and non-gamblers, future research should directly evaluate the link between probability discounting and suboptimal choice in gamblers. If the suboptimal choice task is "diagnostic" for gambling, as Zentall (2014) suggested, there should be a difference in the way gamblers and nongamblers perform, and that difference might be mediated by probability discounting. Additional research is needed to further clarify how humans in general (i.e., with and without pathological gambling habits) perform on the suboptimal choice task, the degree to which their choices are influenced by the presence of signals, and how probability discounting relates to these processes. 


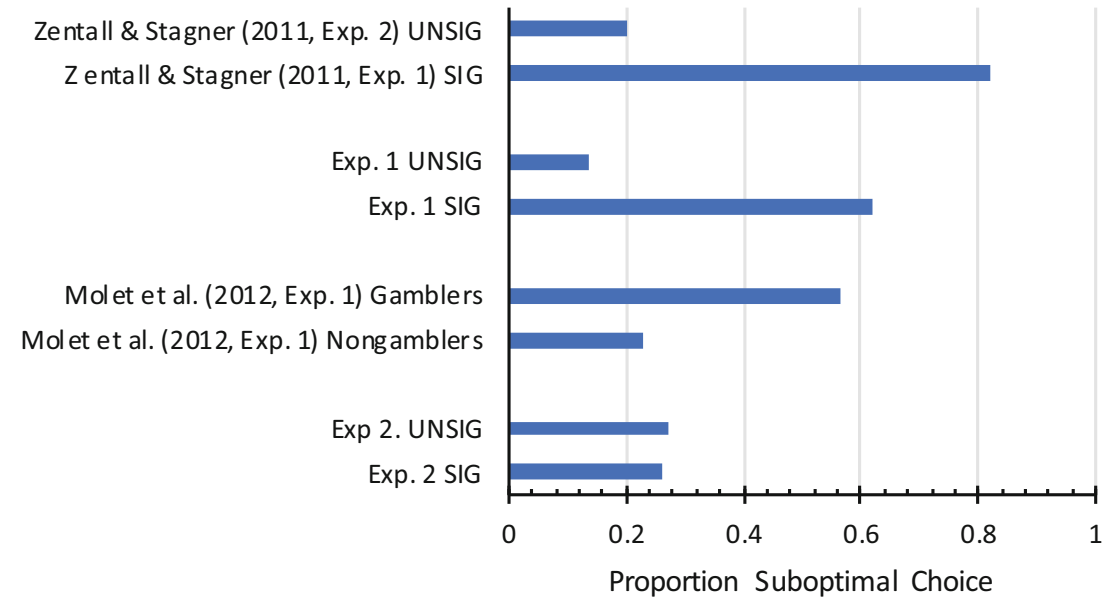

Fig. 6 Mean proportion of suboptimal choices by subjects in Zentall and Stagner (2011, Experiments 1 and 2), Molet et al. (2012, Experiment 1), and Experiments 1 and 2 of the present study. Subjects were pigeons in Zentall and Stagner's study, humans in Molet et al.'s study, and pigeons and humans in Experiments 1 and 2 of the present study, respectively. All three studies used a similar procedure in which a suboptimal alternative

In the suboptimal choice task, differential signals influence the distribution of choice responding, with the potential to decrease the overall amount of food acquired by pigeons. Other procedures have been developed that seek to understand how humans value informative stimuli. For example, Brydevall, Bennett, Murawski, and Bode (2018) assessed the degree to which participants would pay for advance information about a probabilistic outcome. When the information was free, participants chose to receive it. However, as the cost associated with the information increased, participants became less likely to pay for it. This is an interesting finding, and appears to contrast with some of the research that suggests that pigeons engaging in a suboptimal choice task are sometimes unaffected by the probability of food on a suboptimal alternative as long as the outcomes are signaled. For example, Zentall, Laude, Stagner, and Smith (2015) compared an unsignaled alternative that led to food $75 \%$ of the time with a signaled alternative that led to food $50 \%$ of the time in one condition or $25 \%$ of the time in another condition. They found no difference in preference between conditions, despite reducing the probability of food by half on the suboptimal alternative. Different procedures are likely to vary in their sensitivity to particular variables, and it would be useful to develop non-human analogs to procedures like the one used by Brydevall et al. that have effectively been employed with humans, allowing cross-species comparisons from a variety of procedures that measure the impact of information on choice behavior.

Overall, both the results from Molet et al. (2012) and the results reported here show that, not surprisingly, human subjects sometimes engage in suboptimal behavior. Preference for a suboptimal alternative did not occur in either study, as the highest level of preference was observed with regular infrequently led to a large reinforcer and an optimal alternative consistently led to a small reinforcer. In SIG conditions, discriminative stimuli followed choice of the suboptimal alternative and signaled the trial outcome. In UNSIG conditions, stimuli provided following choice of the suboptimal alternative were not differentially associated with the trial outcomes

gamblers (in the study by Molet et al.), and their choice behavior was close to indifference. However, it can be argued that any choice of the suboptimal alternative is suboptimal if one assumes that a subject should always choose the option that provides more reinforcement (but see Vasconcelos, Machado, \& Pandeirada, 2018). Currently, it remains unclear how human performance on the suboptimal choice task is related to gambling, and whether it can be influenced by the same variables that have been shown to influence pigeons' suboptimal choice. The results presented here provide an important initial step in empirically evaluating how humans perform on the suboptimal choice task frequently used with pigeons, and further research is necessary to come to any meaningful conclusions about its usefulness in informing our understanding of gambling behavior.

Author Note We thank the McDaniel College Fall 2016 students in the Psychology of Learning and Lab class for assistance with data collection in Experiment 1 and Alexander Marquis, Mallory Papp, and Rachel Pilver for help with data collection in Experiment 2. We also thank Roger Dunn for programming assistance in Experiment 2.

Some of these data were presented at the 44th Annual Convention of the Association for Behavior Analysis International.

\section{References}

Blanchard, T. C., Hayden, B. Y., \& Bromberg-Martin, E. S. (2015). Orbitofrontal cortex uses distinct codes for different choice attributes in decisions motivated by curiosity. Neuron, 85(3), 602-614. https:// doi.org/10.1016/j.neuron.2014.12.050

Bromberg-Martin, E. S., \& Hikosaka, O. (2009). Midbrain Dopamine neurons signal preference for advance information about upcoming rewards. Neuron, 63(1), 119-126.

Brydevall, M., Bennett, D., Murawski, C., \& Bode, S. (2018). The neural encoding of information prediction errors during non-instrumental 
information seeking. Scientific Reports, 8(1), 6134. https://doi.org/ 10.1038/s41598-018-24566-X.

Cunningham, P. J., \& Shahan, T. A. (2019, May 9). Rats engage in suboptimal choice when the delay to food is sufficiently long. Journal of Experimental Psychology: Animal Learning and Cognition. Advance online publication. https://doi.org/10.1037/ xan0000211

Dunn, R. \& Spetch, M. L. (1990). Choice with uncertain outcomes: Conditioned reinforcement effects. Journal of the Experimental Analysis of Behavior, 53(2), 201-218. https://doi.org/10.1901/jeab. 1990.53-201

Herrnstein, R. J. (1970). On the law of effect. Journal of the Experimental Analysis of Behavior, 13, 243-66.

Kendall, S. B. (1974). Preference for intermittent reinforcement. Journal of the Experimental Analysis of Behavior, 21(3), 463-473. https:// doi.org/10.1901/jeab.1974.21-463

Lalli, J. S., \& Mauro, B. C. (1995). The paradox of preference for unreliable reinforcement: The role of context and conditioned reinforcement. Journal of Applied Behavior Analysis, 28(3), 389-394. https://doi.org/10.1901/jaba.1995.28-389

Lalli, J. S., Mauro, B. C., \& Mace, F. C. (2000). Preference for unreliable reinforcement in children with mental retardation: The role of conditioned reinforcement. Journal of Applied Behavior Analysis, 33(4), 533-544. https://doi.org/10.1901/jaba.2000.33-533

Laude, J. R., Beckmann, J. S., Daniels, C. W., \& Zentall, T. R. (2011). Impulsivity affects suboptimal gambling-like choice by pigeons. Journal of Experimental Psychology: Animal Learning \& Cognition, 40(1), 2-11. https://doi.org/10.1037/xan0000001.

Lesieur, H. R., \& Blume, S. B. (1987). The South Oaks Gambing Screen (SOGS): A new instrument for the identification of pathological gamblers. American Journal of Psychiatry, 144(9), 1184-1188. https://doi.org/10.1176/ajp.144.9.1184

Mazur, J. E. (2007). Species differences between rats and pigeons in choices with probabilistic and delayed reinforcers. Behavioural Processes, 75(2), 220-224. https://doi.org/10.1016/j.beproc.2007. 02.004

McDevitt, M. A, Dunn, R. M., Spetch, M. L., \& Ludvig, E. A. (2016). When good news leads to bad choices. Journal of the Experimental Analysis of Behavior, 105(1), 23-40. https://doi.org/10.1002/jeab. 192

McDevitt, M. A., Pisklak, J. M., Spetch, M. L., \& Dunn, R. M. (2018). The influence of outcome delay on suboptimal choice. Behavioural Processes, 157, 279-285. https://doi.org/10.1016/j.beproc.2018.10. 008

McDevitt, M. A., Spetch, M. L., \& Dunn, R. (1997). Contiguity and conditioned reinforcement in probabilistic choice. Journal of the Experimental Analysis of Behavior, 68(3), 317-327. https://doi. org/10.1901/jeab.1997.68-317

Molet, M., Miller, H. C., Laude, J. R., Kirk, C., Manning, B., \& Zentall, T. R. (2012). Decision making by humans in a behavioral task: Do humans, like pigeons, show suboptimal choice? Learning \& Behavior, 40(4), 439-447. https://doi.org/10.3758/s13420-0120065-7

Myerson, J., Green, L., \& Warusawitharana, M. (2001). Area under the curve as a measure of discounting. Journal of the Experimental Analysis of Behavior, 76(2), 235-243. https://doi.org/10.1901/jeab. 2001.76-235
Peirce, J. W. (2007). PsychoPy - Psychophysics software in Python. Journal of Neuroscience Methods, 162(1-2), 8-13. https://doi.org/ 10.1016/j.jneumeth.2006.11.017

Petry, N. (2012). Discounting of probabilistic rewards is associated with gambling abstinence in treatment-seeking pathological gamblers. Journal of Abnormal Psychology, 121(1), 151-159. https://doi.org/ 10.1037/a0024872

Rachlin, H., Raineri, A., \& Cross, D. (1991). Subjective probability and delay. Journal of the Experimental Analysis of Behavior, 55(2), 233244. https://doi.org/10.1901/jeab.1991.55-233

Smith, T. R., Beran, M. J., \& Young, M. E. (2017). Gambling in rhesus macaques (Macaca mulatta): The effect of cues signaling risky choice outcomes. Learning \& behavior, 45(3), 288-299. https:// doi.org/10.3758/s13420-017-0270-5

Spetch, M., Belke, T., Barnet, R., Dunn, R., \& Pierce, W. (1990). Suboptimal choice in a percentage-reinforcement procedure: Effects of signal condition and terminal-link length. Journal of the Experimental Analysis of Behavior, 53(2), 219-234. https://doi.org/ 10.1901/jeab.1990.53-219.

Spetch, M. L., Mondloch, M., Belke, T., \& Dunn, R. (1994). Determinants of pigeons' choice between certain and probabilistic outcomes. Animal Learning \& Behavior, 22, 239-251. https://doi. org/10.3758/BF03209832

Trujano, R. E., \& Orduña, V. (2015). Rats are optimal in a choice task in which pigeons are not. Behavioural Processes, 119, 22-27.

Vasconcelos, M., Monteiro, T., \& Kacelnik, A. (2015). Irrational choice and the value of information. Scientific Reports, 5, 13874. Retrieved from https://doi.org/10.1038/srep13874

Vasconcelos, M., Machado, A., \& Pandeirada, J. N. S. (2018). Ultimate explanations and suboptimal choice. Behavioural Processes, 152, 63-72.

Zentall, T. R. (2014). Suboptimal choice by pigeons: An analog of human gambling behavior. Behavioral Processes, 103, 156-164. https://doi. org/10.1016/j.beproc.2013.11.0004

Zentall, T. R. (2016a). An animal model of human gambling. International Journal of Psychological Research, 9(2), 96-112.

Zentall, T. R. (2016b). Resolving the paradox of suboptimal choice. Journal of Experimental Psychology: Animal Learning and Cognition, 1-14. https://doi.org/10.1037/xan0000085

Zentall, T. R., \& Laude, J. R. (2013). Do pigeons gamble? I wouldn't bet against it. Current Directions in Psychological Science, 22(4), 271277. https://doi.org/10.1177/0963721413480173

Zentall, T. R., Laude, J. R., Stagner, J. P., \& Smith, A. P. (2015). Suboptimal choice by pigeons: Evidence that the value of the conditioned reinforcer rather than its frequency determines choice. The Psychological Record, 65(2), 223-229. https://doi.org/10.1007/ s40732-015-0119-2

Zentall, T. R., \& Stagner, J. (2011). Maladaptive choice behaviour by pigeons: An animal analogue and possible mechanism for gambling (sub-optimal human decision-making behaviour). Proceedings $B$ : Biological Sciences / The Royal Society, 278, 1203-1208. https:// doi.org/10.1098/rspb.2010.1607

Publisher's note Springer Nature remains neutral with regard to jurisdictional claims in published maps and institutional affiliations. 